\title{
SGIMOBS: the million minds approach revisited in mobile context
}

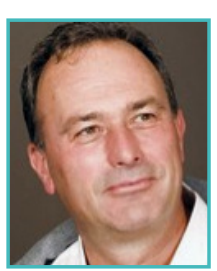

Barend Mons is Professor of Biosemantics at the LUMC and is one of the scientific directors of $\mathrm{NBIC}$, the Netherlands Bioinformatics Center. In addition he acts as a Life Sciences 'eScience integrator' in the Netherlands eScience centre. Currently, he coordinates the creation of the Data Integration and Stewardship Centre (DISCELIXIR) and in that capacity he is also the head of Node of the developing Dutch node in the ELIXIR ESFRI project.

Barend Mons is a molecular biologist by training and received his PhD on genetic differentiation of malaria parasites from Leiden University (1986). He performed over a decade of research on malaria genetics and vaccine development, also serving for 3 years the research department of the European Commission in this field. He did gain further experience in science management at the Research council of The Netherlands (NWO).

Barend is the co founder of three spin-off companies in biotechnological and semantic technologies and is an advisor for several companies as well. From the year 2000 onward he increasingly focuses on the development of semantic technologies to manage big data and he founded the Biosemantics groups, first at Erasmus University in Rotterdam and later also in Leiden. Both groups collaborate very closely.

His research is currently focused on nanopublications as a substrate for in silico knowledge discovery. Barend is also one of the founders of the Concept Web Alliance, with "nanopublications" as its first brainchild. Nanopublications are currently implemented in the semantic project of the Innovative Medicines Initiative (IMI) called Open PHACTS.

In this talk, he will revisit the 'need to engage a million minds in expert crowd sourcing' based on his highly cited 2008 paper "Mons et al. Calling on a million minds for community annotation in WikiProteins, Genome Biology 2008, 9:R89 ".

Now that mobile technology is so much further in development and social acceptance, and the first real scientific applications are coming on the market, there is a need to revisit how best to engage people in expert crowdsourcing. 